\title{
Using air cholangiography to reduce postendoscopic retrograde cholangiopancreatography cholangitis in patients with malignant hilar obstruction
}

\author{
Qi-Bin He ${ }^{1}$, Ru-Hua Zheng ${ }^{2}$, Yi Wang ${ }^{2}$, Lei Wang ${ }^{2}$, Lu-Xuan Tan ${ }^{3}$, Gui-Xia Meng ${ }^{3}$, Huan Zhong ${ }^{3}$, \\ Jie Duan", Ai-Dong Gu ${ }^{4}$ \\ ${ }^{1}$ Department of Gastroenterology, Nanjing Jiangning Hospital, The Affiliated Jiangning Hospital of Nanjing Medical University, Nanjing, China; \\ ${ }^{2}$ Department of Gastroenterology, Nanjing Drum Tower Hospital Clinical College of Nanjing Medical University, Nanjing, China; ${ }^{3}$ Department \\ of Gastroenterology, The Second Hospital of Nanjing, Nanjing University of Chinese Medicine, Nanjing, China; ${ }^{4}$ Department of Hepatobiliary \\ Surgery, The Second Hospital of Nanjing, Nanjing University of Chinese Medicine, Nanjing, China
}

Contributions: (I) Conception and design: QB He, AD Gu, L Wang; (II) Administrative support: GX Meng; (III) Provision of study materials or patients: J Duan; (IV) Collection and assembly of data: RH Zheng, Y Wang; (V) Data analysis and interpretation: LX Tan; (VI) Manuscript writing: All authors; (VII) Final approval of manuscript: All authors.

Correspondence to: Lei Wang, MD. Department of Gastroenterology, Nanjing Drum Tower Hospital Clinical College of Nanjing Medical University, No. 321 of Zhongshan Road, Gulou District, Nanjing 210009, China. Email: drwangleiga@163.com; Ai-Dong Gu, MM. Department of Hepatobiliary Surgery, The Second Hospital of Nanjing, Nanjing University of Chinese Medicine, No. 1-1 Zhongfu Road, Gulou District, Nanjing 210003, China. Email: adguaidong@163.com.

Background: Cholangitis after endoscopic retrograde cholangiopancreatography (ERCP) is a major problem for patients with hilar biliary obstruction. To date, it remains unclear whether air-contrast cholangiography (ACC) can reduce cholangitis in these patients. For this reason, our study assesses the efficacy of reducing cholangitis through ACC.

Methods: This paper presents a retrospective study conducted at a tertiary university hospital. We enrolled patients who were diagnosed with hilar structures and underwent ERCP between January 2012 and December 2018. From 2015 onwards, ACC was performed following the successful selective cannulation into the dilated intrahepatic bile duct of these patients. The primary aim was to assess patients with cholangitis in both an ACC group and iodine contrast cholangiography (ICC) group.

Results: This study included 80 patients, 35 of whom received ACC and 45 who received ICC. There were no differences between the 2 groups in terms of the number of patients who underwent endoscopic papillotomy, endoscopic nasobiliary drainage, endoscopic biliary stent placement, or other technical procedures or complications. A total of 19 patients (23.8\%) presented with fever (cholangitis) after the ERCP procedure (4 ACC, 15 ICC; $11.4 \%$ vs. 33.3\%, respectively; $\mathrm{P}=0.03$ ). One patient in the ICC group who obtained a plastic stent for palliative drainage died 2 weeks post-ERCP. Among the other 18 cholangitis patients, 8 (1 ACC, 7 ICC) were treated with additional ERCP or percutaneous transhepatic biliary drainage (PTBD), while the remaining 10 only received antibiotics. One patient in the ICC group who obtained a plastic stent for palliative drainage died 2 weeks post-ERCP.

Conclusions: We found that ACC significantly reduced the incidence of cholangitis in patients with hilar obstruction.

Keywords: Perihilar cholangiocarcinoma (PHC); endoscopic retrograde cholangiopancreatography (ERCP); air contrast cholangiography; endoscopic biliary stenting (EBS); endoscopic nasobiliary drainage (ENBD)

Submitted Apr 29, 2021. Accepted for publication Nov 02, 2021.

doi: 10.21037 /qims-21-462

View this article at: https://dx.doi.org/10.21037/qims-21-462 


\section{Introduction}

Perihilar cholangiocarcinoma (PHC) is an uncommon malignancy with a poor prognosis that accounts for more than $60 \%$ of bile duct carcinomas (1-3). Although doubt still exists around the effectiveness of preoperative biliary drainage in patients with malignant biliary obstruction, it has been shown that preoperative drainage reduces jaundice and bacterial translocation, improves liver function and nutritional status, and enhances the ability of the liver to regenerate postoperatively (4). Despite controversy concerning the 2 primary drainage approaches, which include endoscopic retrograde cholangiopancreatography (ERCP) and percutaneous transhepatic biliary drainage (PTBD), studies have concluded that ERCP is more suitable than is PTBD in terms of compliance among perihilar tumor patients (5-7). In the case of inoperable hilar biliary obstruction, ERCP is one of the major palliative methods for treating obstructive jaundice or cholangitis. Biliary drainage not only relieves jaundice and associated pruritus but improves related symptoms such as anorexia, diarrhea, and disturbed sleeping patterns. It can also enhance a patient's quality of life $(1,8)$.

In this study, we found post-ERCP cholangitis to be a major problem for hilar biliary obstruction patients. It has been reported that early cholangitis increases when both lobes are opacified using contrast (diluted iodine contrast agent), but only if 1 is drained (9). The present study performed both air contrast cholangiography (ACC) and the more established iodine contrast cholangiography (ICC) to reduce post-ERCP cholangitis. We present the following article in accordance with the Strengthening the Reporting of Observational Studies in Epidemiology (STROBE) reporting checklist (available at https://dx.doi.org/10.21037/ qims-21-462).

\section{Methods}

\section{Study design}

This retrospective study was performed at a tertiary university hospital that treats more than 1,500 cases of ERCP annually. In the past, conventional cholangiography had been performed using an iodine contrast, but starting in 2015, we began using air as a contrast for performing cholangiography for hilar structures. The technical and clinical characteristics between ACC and ICC were assessed, and the endoscopic data and clinical presentation of patients who underwent endoscopic biliary drainage of hilar structures were collected.

\section{Patients}

This study was conducted in accordance with the Declaration of Helsinki (as revised in 2013). It was approved by the Ethics Committee of Nanjing Drum Tower Hospital of the Nanjing University Medical School (Nanjing, China). Written informed consent for inclusion in the study was obtained from each patient prior to undergoing ERCP.

Our study screened for patients who, from January 2012 to December 2018, had been diagnosed with hilar structures including PHC, gallbladder carcinoma, hepatic cancer, and metastatic tumors. Patients who underwent preoperative or palliative ERCP drainage were enrolled. Patients who underwent PTBD or received self-expandable metallic stent (SEMS) placement as an initial drainage procedure were excluded as were patients who received emergency ERCP.

\section{The ERCP procedure}

All ERCP procedures were carried out by 6 experienced endoscopists who had cumulatively performed more than 1,000 ERCPs in the past. Preprocedure, computed tomography, and magnetic resonance cholangiopancreatography (MRCP) specifics were obtained for all the included patients and were used to confirm the diagnosis and grade of hilar block. This information was also used to preselect the intended lobe or segment for drainage (based on the liver volume being drained and ductal dilatation). Prophylactic intravenous antibiotics (third generation cephalosporins) were initiated 1 hour before the procedure and continued for 3 days. The ERCP was performed using the standard procedure noted in our previous paper (10). After bile duct cannulation had been successfully performed, a hydrophilic guidewire (Radifocus Guide Wire M, RF-GA35263M, TERUMO, Japan) was advanced through the malignant stenosis into the preselected intrahepatic dilated duct based on MRCP under fluoroscopic guidance. A papillotome was then passed over the guidewire across the stenosis until bile was aspirated (minimum $5-10 \mathrm{~mL}$ ) to confirm the position and to decrease the intraductal pressure. ACC was then performed by injecting 10 to $15 \mathrm{~mL}$ of air, and the opacified ductal system was compared with the segment intended for drainage using MRCP images. If the guidewire failed to pass through the structure into the dilated duct, iodine contrast was injected to obtain a roadmap (these patients were then 

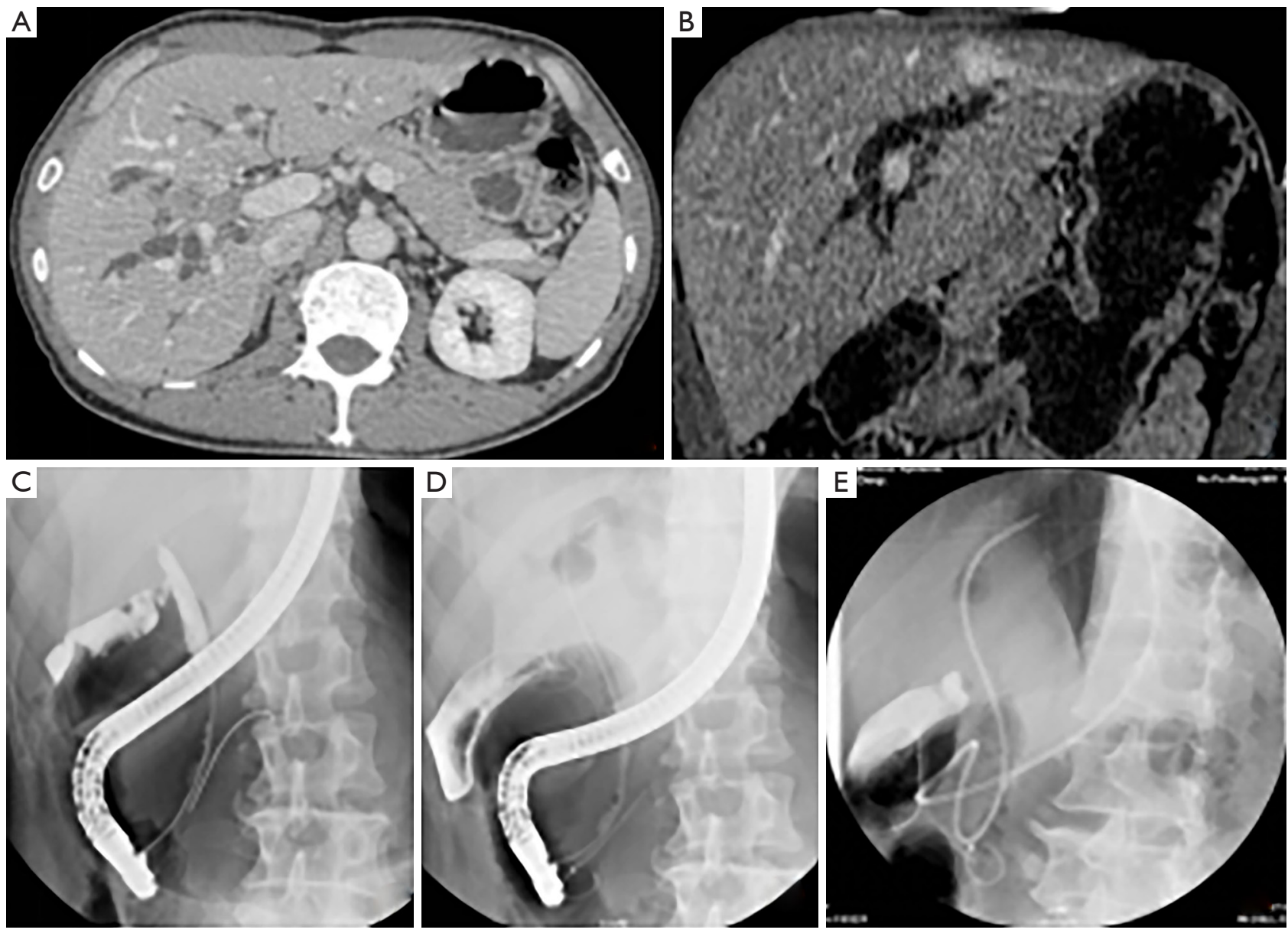

Figure 1 Single ENBD preoperative drainage for PHC. This patient, a 66-year-old male, had Bismuth type IIIa strictures caused by hilar cholangiocarcinoma. His initial serum bilirubin level increased to $12.2 \mathrm{mg} / \mathrm{dL}$. One 7-Fr ENBD catheter was inserted into the left lateral bile duct (future remnant lobe), and the externally drained bile was replaced by a nasointestinal tube. His serum bilirubin level decreased to $2.0 \mathrm{mg} / \mathrm{dL}$ at 42 days post-ENBD, and he had undergone segments 1, 5-8 hepatectomy. (A) Axial computed tomography with contrast shows the mass in the right liver. (B) Coronal computed tomography shows dilated left bile duct. (C) Cholangiography contrast in the common bile duct shows the distal part of the structure. (D) Air cholangiography of the left intrahepatic duct. (D) ENBD drainage for left intrahepatic duct. (E) ENBD drainage for left intrahepatic duct. ENBD, endoscopic nasobiliary drainage; PHC, perihilar cholangiocarcinoma.

enrolled into the ICC group). A cytology brush (Cytomax II, Cook Medical LLC, Bloomington, IN, USA) was then used for cytopathologic examination. After the targeted bile duct was cannulated, an endoscopic nasobiliary drainage (ENBD) tube (with or without plastic stent) was placed in the bile duct. The stents used were 7- and 8.5-Fr biliary plastic stents, and the drainage tube was a 7-Fr ENBD tube. In our institution, we prefer a unilateral ENBD to the future lobe as an initial preoperative drainage procedure with hilar cholangiocarcinoma (Figure 1), a method that is accepted in Japan $(7,11)$. For palliative treatment, a plastic stent (with or without ENBD) was implanted in the dilated bile duct (Figure 2).

\section{Patient follow-up}

After completing the ERCP procedure, patients were admitted to a ward for at least 1 week. Their symptoms, physical signs, amylase, full blood picture, and blood biochemistry were recorded. The candidates for curative surgery were admitted until their surgery date. For palliative drainage, if the total bilirubin level decreased more than 20\%, the ENBD was removed or another ERCP was performed to replace the ENBD catheter with a plastic 

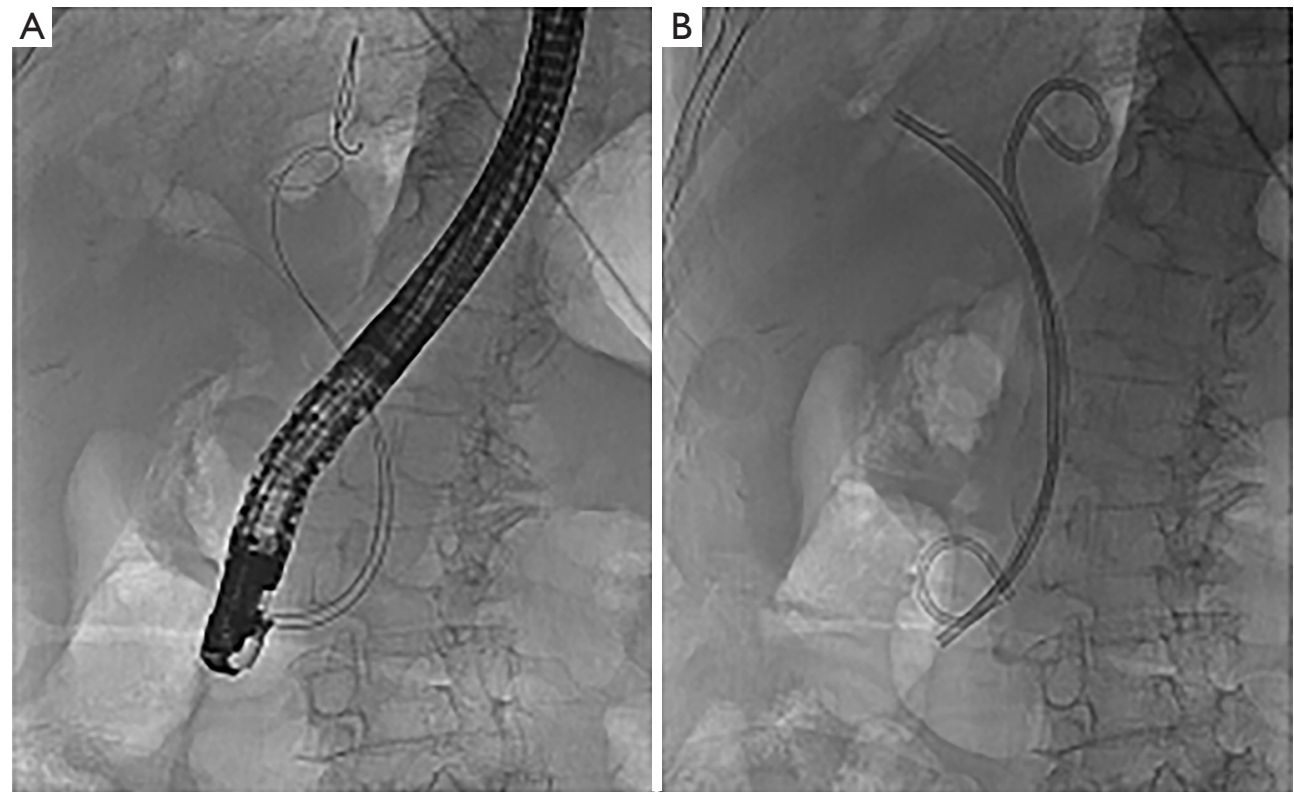

Figure 2 Palliative drainage for perihilar cholangiocarcinoma (Bismuth type IV). (A) Air cholangiogram of the right and left intrahepatic duct. (B) Plastic stents were inserted into the right and left intrahepatic duct.

stent. After being discharged, patients were followed up in the hospital's outpatient clinic after 1 month. If the patient presented with cholangitis, additional ERCP or PTBD was performed if necessary. Considering cholangitis is directly related to the ERCP operation due to poor retention and drainage of the intrahepatic bile duct with the contrast agent (bacteria introduced), we only conducted a 1-month follow-up.

\section{Definitions}

Cholangitis is considered to be present in patients who present with the onset of fever (a temperature higher than $38^{\circ} \mathrm{C}$ ) and elevated total bilirubin, with or without septic shock syndromes (e.g., hypotension and tachycardia). The diagnostic criteria and severity classification criteria of the Tokyo Cholangitis Guidelines are not applicable to the diagnosis and classification of postoperative cholangitis. Post-ERCP pancreatitis (PEP) and post-ERCP bleeding and perforation were defined by consensus criteria as noted in our previous studies $(10,12)$.

\section{Outcomes}

The primary end point was cholangitis in the ACC and ICC groups. Secondary end point included the success rate of ERCP and its related complications.

\section{Statistical analysis}

In our study, quantitative variables are described as mean \pm standard deviation (SD), and a Student's $t$-test and MannWhitney $U$ test were used if the variables did not conform to normal distribution. Qualitative variables are presented as numbers and were compared by Pearson's chi-square test. All tests were two-tailed, and $\mathrm{P}$ values smaller than 0.05 were considered statistically significant. All statistical analyses were performed using SPSS Statistics 23 software (IBM Corporation, Armonk, NY, USA).

\section{Results}

\section{Patients}

During the study period, 141 patients were admitted for biliary evaluation and drainage. Among them, 130 were diagnosed with PHC. Sixty-one patients were excluded for the following reasons: initial PTBD $(n=48)$, placement of metal stent $(n=3)$, emergency ERCP $(n=3)$, and previous drainage $(n=7)$. Two ERCP procedures were unsuccessful due to the failure of selective cannulation of the future remnant lobe and were converted to PTBD. Subsequently, a 
total of 80 patients were included in this study, 35 of whom received ACC, and 45 who received ICC (Figure 3). Prior to 2015, 27 procedures were performed, 3 of which included cholangiography with ACC and 24 with ICC. After

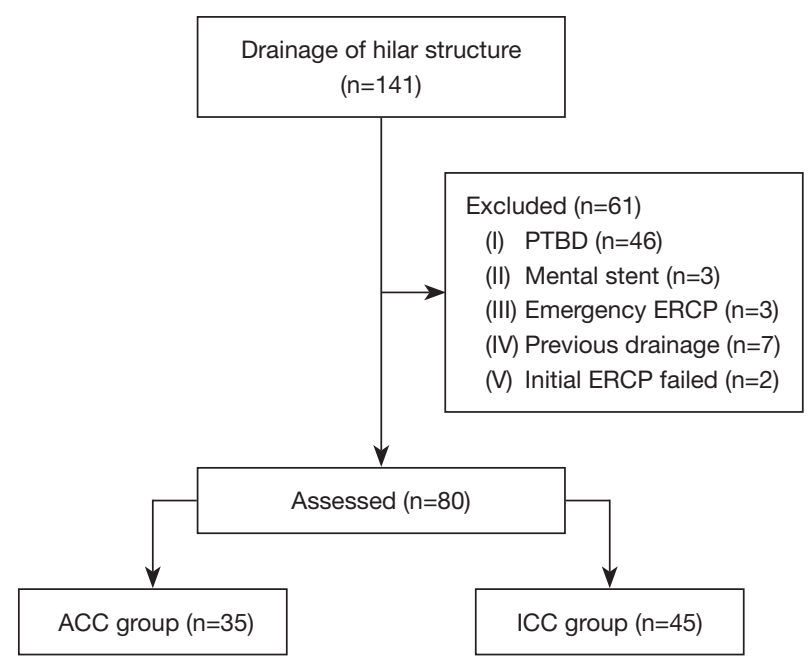

Figure 3 Flow diagram. PTBD, percutaneous transhepatic biliary drainage; ACC, air contrast cholangiography; ICC, iodine contrast cholangiography.
2015, 53 procedures were performed, 32 with ACC and 21 with ICC. Among the 80 patients, 45 were scheduled for preoperative drainage and the remaining 35 were scheduled for palliative drainage.

The demographic parameters and purpose for ERCP did not significantly differ between the 2 groups (Table 1). Of the 35 patients in the ACC group, 1 (2.9\%) was diagnosed with Bismuth-Corlette (B-C) type I, and 8 (22.9\%), $14(40.0 \%)$, and $12(34.3 \%)$ were diagnosed with B-C type II, III, and IV, respectively. The tumors in the ICC group were classified as B-C type I ( $\mathrm{n}=0)$, II $(\mathrm{n}=7)$, III $(\mathrm{n}=20)$, and IV $(\mathrm{n}=18)$. There were no significant differences between the ACC and ICC groups (Table 1). The initial serum total bilirubin level in the ACC group was $12.4 \pm 7.3 \mathrm{mg} / \mathrm{dL}$, which was comparable to the findings for the ICC group $(\mathrm{P}>0.05)$.

\section{Technical characteristics}

Table 2 shows the technical characteristics of the ACC and ICC groups. There were no differences between the 2 groups in terms of the number of patients who underwent endoscopic sphincterotomy (EPT), ENBD, and endoscopic biliary stenting (EBS); or in the remaining technical characteristics.

Table 1 Demographics and indications for ERCP

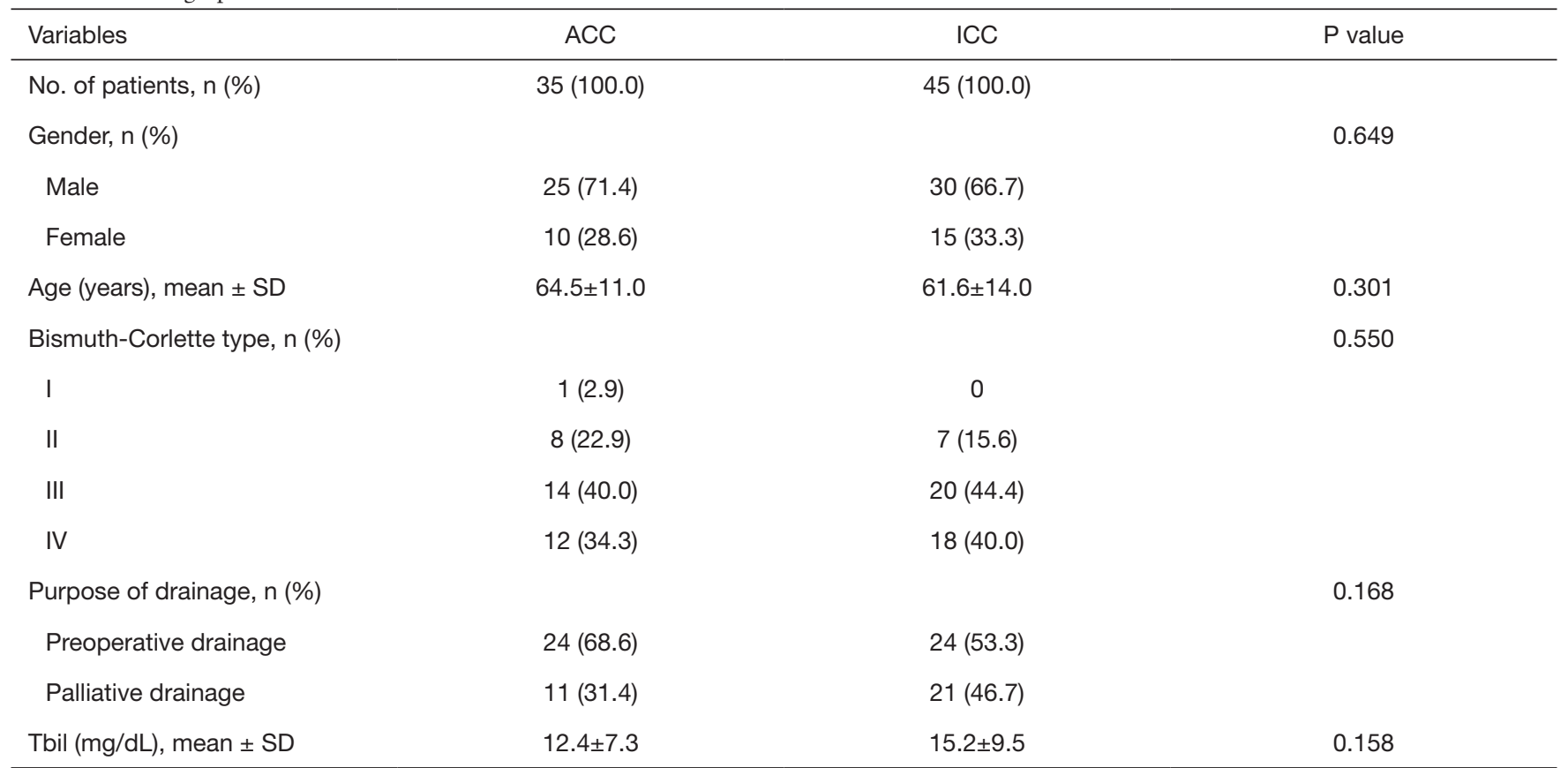

ACC, air contrast cholangiography; ICC, iodine contrast cholangiography; ERCP, endoscopic retrograde cholangiopancreatography; SD, standard deviation; Tbil, total bilirubin level. 
Table 2 Technical characteristics

\begin{tabular}{lccc}
\hline Characteristics & ACC, $\mathrm{n}(\%)$ & ICC, $\mathrm{n}(\%)$ & P value \\
\hline EPT & $17(48.6)$ & $24(53.3)$ & 0.822 \\
ENBD & $29(82.9)$ & $40(88.9)$ & 0.521 \\
EBS & $11(31.4)$ & $19(42.2)$ & 0.360 \\
PDGA & $13(37.1)$ & $15(33.3)$ & 0.815 \\
PDS & $14(40.0)$ & $15(33.3)$ & 0.641 \\
Rectal indomethacin & $6(17.1)$ & $3(6.7)$ & 0.169 \\
\hline
\end{tabular}

ACC, air contrast cholangiography; ICC, iodine contrast cholangiography; EPT, endoscopic Sphincterotomy; ENBD, endoscopic nasobiliary drainage; EBS, endoscopic biliary stent; PDGA, pancreatic duct guidewire assistant cannulation; PDS, pancreatic duct stent.

Table 3 Clinical presentation and adverse events

\begin{tabular}{lccc}
\hline Variables & ACC, n (\%) & ICC, n (\%) & P value \\
\hline PEP & $2(5.7)$ & $9(20.0)$ & 0.101 \\
Cholangitis & $4(11.4)$ & $15(33.3)$ & 0.033 \\
Bleeding & $2(5.7)$ & $2(4.4)$ & 1.000 \\
Perforation & 0 & 0 & - \\
Death & 0 & $1(2.2)$ & 1.000 \\
Tbil improvement $(\mathrm{mg} / \mathrm{dL})^{*}$, mean \pm SD & $0.43 \pm 0.45$ & $0.47 \pm 0.36$ & 0.680 \\
\hline
\end{tabular}

*, Tbil improvement was presented with the decrease of total bilirubin everyday. PEP, post-ERCP pancreatitis; ACC, air contrast cholangiography; ICC, iodine contrast cholangiography; Tbil, total bilirubin level; ERCP, endoscopic retrograde cholangiopancreatography; $\mathrm{SD}$, standard deviation.

\section{Outcomes and adverse events}

Two patients ( 1 in the ACC group and 1 in the ICC group) did not complete a follow-up after being discharged. One patient in the ICC group, who obtained a plastic stent for palliative drainage, died 2 weeks post-ERCP (initial total bilirubin level $50.5 \mathrm{mg} / \mathrm{dL}$ ). Table 3 shows the total bilirubin level improvement and the incidence rate of overall complications in the 2 groups, including PEP, cholangitis, hemorrhage, and perforation. In total, 19 patients $(23.8 \%)$ presented with cholangitis following the ERCP procedure (4 ACC, 15 ICC; $11.4 \%$ vs. $33.3 \%$; $\mathrm{P}=0.03$ ). One patient in the ICC group, who obtained a plastic stent for palliative drainage, was diagnosed cholangitis and died 2 weeks postERCP (initial total bilirubin level $50.5 \mathrm{mg} / \mathrm{dL}$ ). Among the other 18 patients with cholangitis, 8 (1 ACC, 7 ICC) were treated with additional ERCP or PTBD, while the remaining 10 only received antibiotics. For the 8 patients who received additional ERCP or PTDB, the location was the other intrahepatic bile duct (4 cases, including the 1
ACC), the other segment ( 2 cases), and the original drainage location ( 2 cases). The total bilirubin level decreased more than $50 \% 1$ month after the ERCP procedure for $30 \mathrm{ACC}$ and 38 ICC patients. The rate of decline in the ACC group and ICC group was $0.43 \pm 0.45$ and $0.47 \pm 0.36 \mathrm{mg} / \mathrm{dL}$, respectively $(\mathrm{P}>0.05)$. Two patients in each group developed post-sphincterotomy bleeding, 1 of whom (in the ACC group) was diagnosed as moderate in this regard with a blood transfusion requirement and endoscopic hemostasis. A total of 11 patients $(13.8 \%)$ met the criteria of mild PEP, including 2 in the ACC group and 9 in the ICC group. There was no statistical difference in the PEP rates between the ACC and ICC groups $(\mathrm{P}=0.10)$.

\section{Discussion}

Malignant hilar obstruction requires a more challenging endoscopic drainage operation than do other types of ERCP. Cholangitis is the most common post-ERCP complication and occurred in $36-65 \%$ of cases reported in 
a recent meta-analysis (13). One of the main risk factors of post-ERCP cholangitis is inadequate drainage of all obstructed intrahepatic ducts (14). It is important for endoscopists to avoid filling all intrahepatic segments and to drain all segments that are filled with contrast in the case of malignant hilar strictures. Fluid contrast can easily introduce bacteria into the intrahepatic duct, and it can be technically difficult to completely drain all segments filled with contrast. It was reported that air, rather than the typical iodine contrast, can be used as a contrast for cholangiograms and can reduce the incidence of cholangitis when a metal stent is being placed $(15,16)$. Accordingly, we hypothesize that ACC may be applicable for PHC patients receiving ENBD and EBS drainage. Our study showed that ACC was significantly associated with a lower incidence of postERCP cholangitis in the ENBD and EBS for malignant hilar biliary obstruction patients $(11.4 \%$ vs. $33.3 \%$; $\mathrm{P}<0.05)$. In other words, we found that the rate of cholangitis was significantly reduced in the ACC group of our study. Based on our experience, the main reason for these results were as follows: selective cannulation into the dilated intrahepatic bile duct was followed by ACC, which enabled better implantation of the ENBD (particularly in our preoperative group), and assisted ENBD/EBS placement was conducted with minimal intervention and procedure duration. Two prospective randomized controlled studies have found similar results. Sud et al. (17) found that using ACC was as safe and as effective as dye cholangiography in patients with malignant hilar biliary obstruction, and it decreased the risk of post-ERCP cholangitis ( $4 \%$ vs. $16.6 \%$; $\mathrm{P}<0.05$ ). In the study of Zhang et al. (18), they also confirmed that ACC can reduce the incidence of cholangitis $(5.6 \% \mathrm{vs}$. $33.3 \%, \mathrm{P}=0.04)$. In our study, 8 patients were treated with additional ERCP or PTBD (1 ACC, 7 ICC). Since there was only 1 ACC patient, we were unfortunately unable to further refine the analysis.

Kawashima et al. (19) also reported that the only significant risk factor associated with post-ENBD cholangitis was if EPT had been previously conducted. In their study, the incidence of cholangitis was $43.8 \%$ (21/48 patients). In our study, only minor EPT was performed to prevent PEP and to assist in inserting the stent and/or ENBD catheter.

Air embolism is a rare but lethal complication of air insufflation during ERCP. However, no cases of air embolism have been directly associated with ACC (20). To the best of our knowledge, no reports have been published to date denoting the occurrence of an air embolism in an
ACC procedure (15-17).

It is important to note that this study also included some limitations: it was a retrospective study that included a small sample size, the follow-up period was only 1 month, and we did not report the overall patient survival rates. The efficacy and safety of ACC should thus be further investigated in prospective multicenter studies.

\section{Conclusions}

In summary, ACC significantly reduced the incidence of cholangitis in patients with malignant hilar obstruction. Accordingly, the use of this procedure deserves further consideration and evaluation.

\section{Acknowledgments}

Funding: None.

\section{Footnote}

Reporting Checklist: The authors have completed the STROBE reporting checklist. Available at https://dx.doi. org/10.21037/qims-21-462

Conflicts of Interest: All authors have completed the ICMJE uniform disclosure form (available at https://dx.doi. org/10.21037/qims-21-462). The authors have no conflicts of interest to declare.

Ethical Statement: The authors are accountable for all aspects of the work in ensuring that questions related to the accuracy or integrity of any part of the work are appropriately investigated and resolved. This study was conducted in accordance with the Declaration of Helsinki (as revised in 2013). This study was conducted with approval from the Ethics Committee of The Second Hospital of Nanjing, Nanjing University of Chinese Medicine. Written informed consent was obtained from all participants.

Open Access Statement: This is an Open Access article distributed in accordance with the Creative Commons Attribution-NonCommercial-NoDerivs 4.0 International License (CC BY-NC-ND 4.0), which permits the noncommercial replication and distribution of the article with the strict proviso that no changes or edits are made and the original work is properly cited (including links to both the formal publication through the relevant DOI and the license). 
See: https://creativecommons.org/licenses/by-nc-nd/4.0/.

\section{References}

1. Singhal D, van Gulik TM, Gouma DJ. Palliative management of hilar cholangiocarcinoma. Surg Oncol 2005;14:59-74.

2. Geller A. Klatskin tumor--palliative therapy: the jury is still out or may be not yet in... . Gastrointest Endosc 2009;69:63-5.

3. Nagino M, Ebata T, Yokoyama Y, Igami T, Sugawara G, Takahashi Y, Nimura Y. Evolution of surgical treatment for perihilar cholangiocarcinoma: a single-center 34-year review of 574 consecutive resections. Ann Surg 2013;258:129-40.

4. van der Gaag NA, Kloek JJ, de Castro SM, Busch OR, van Gulik TM, Gouma DJ. Preoperative biliary drainage in patients with obstructive jaundice: history and current status. J Gastrointest Surg 2009;13:814-20.

5. Kim KM, Park JW, Lee JK, Lee KH, Lee KT, Shim SG. A Comparison of Preoperative Biliary Drainage Methods for Perihilar Cholangiocarcinoma: Endoscopic versus Percutaneous Transhepatic Biliary Drainage. Gut Liver 2015;9:791-9.

6. Jo JH, Chung MJ, Han DH, Park JY, Bang S, Park SW, Song SY, Chung JB. Best options for preoperative biliary drainage in patients with Klatskin tumors. Surg Endosc 2017;31:422-9.

7. Kawakami H, Kuwatani M, Onodera M, Haba S, Eto K, Ehira N, Yamato H, Kudo T, Tanaka E, Hirano S, Kondo $\mathrm{S}$, Asaka $\mathrm{M}$. Endoscopic nasobiliary drainage is the most suitable preoperative biliary drainage method in the management of patients with hilar cholangiocarcinoma. J Gastroenterol 2011;46:242-8.

8. Soehendra N, Reynders-Frederix V. Palliative bile duct drainage - a new endoscopic method of introducing a transpapillary drain. Endoscopy 1980;12:8-11.

9. Chang WH, Kortan P, Haber GB. Outcome in patients with bifurcation tumors who undergo unilateral versus bilateral hepatic duct drainage. Gastrointest Endosc 1998;47:354-62.

10. He Q, Wang L, Peng C, Zou X, Zhan Q, Xu Y, Liu Q, Qian J, Gong L, Shen Y, Chen J. Modified prophylactic 5 -fr pancreatic duct stent enhances the rate of spontaneous dislodgement: A multicenter randomized controlled trial. United European Gastroenterol J 2018;6:1519-26.

11. Kawakubo K, Kawakami H, Kuwatani M, Haba S, Kudo T, Taya YA, Kawahata S, Kubota Y, Kubo K, Eto K, Ehira N, Yamato H, Onodera M, Sakamoto N. Lower incidence of complications in endoscopic nasobiliary drainage for hilar cholangiocarcinoma. World J Gastrointest Endosc 2016;8:385-90.

12. He QB, Xu T, Wang J, Li YH, Wang L, Zou XP. Risk factors for post-ERCP pancreatitis and hyperamylasemia: A retrospective single-center study. J Dig Dis 2015;16:471-8.

13. Tang Z, Yang Y, Meng W, Li X. Best option for preoperative biliary drainage in Klatskin tumor: A systematic review and meta-analysis. Medicine (Baltimore) 2017;96:e8372.

14. Deviere J, Baize M, de Toeuf J, Cremer M. Long-term follow-up of patients with hilar malignant stricture treated by endoscopic internal biliary drainage. Gastrointest Endosc 1988;34:95-101.

15. Sud R, Puri R, Hussain S, Kumar M, Thawrani A. Air cholangiogram: a new technique for biliary imaging during ERCP. Gastrointest Endosc 2010;72:204-8.

16. Lee JM, Lee SH, Jang DK, Chung KH, Park JM, Paik WH, Lee JK, Ryu JK, Kim YT. Air cholangiography in endoscopic bilateral stent-in-stent placement of metallic stents for malignant hilar biliary obstruction. Therap Adv Gastroenterol 2016;9:189-98.

17. Sud R, Puri R, Choudhary NS, Mehta A, Jain PK. Air cholangiogram is not inferior to dye cholangiogram for malignant hilar biliary obstruction: a randomized study of efficacy and safety. Indian J Gastroenterol 2014;33:537-42.

18. Zhang R, Zhao L, Liu Z, Wang B, Hui N, Wang X, Huang R, Luo H, Fan D, Pan Y, Guo X. Effect of $\mathrm{CO} 2$ cholangiography on post-ERCP cholangitis in patients with unresectable malignant hilar obstruction - a prospective, randomized controlled study. Scand J Gastroenterol 2013;48:758-63.

19. Kawashima H, Itoh A, Ohno E, Itoh Y, Ebata T, Nagino M, Goto H, Hirooka Y. Preoperative endoscopic nasobiliary drainage in 164 consecutive patients with suspected perihilar cholangiocarcinoma: a retrospective study of efficacy and risk factors related to complications. Ann Surg 2013;257:121-7.

20. Ali Z, Bolster F, Goldberg E, Fowler D, Li L. Systemic air embolism complicating upper gastrointestinal endoscopy: a case report with post-mortem CT scan findings and review of literature. Forensic Sci Res 2017;1:52-7.

Cite this article as: $\mathrm{He} \mathrm{QB}$, Zheng $\mathrm{RH}$, Wang $\mathrm{Y}$, Wang $\mathrm{L}$, Tan LX, Meng GX, Zhong H, Duan J, Gu AD. Using air cholangiography to reduce postendoscopic retrograde cholangiopancreatography cholangitis in patients with malignant hilar obstruction. Quant Imaging Med Surg 2022;12(3):1698-1705. doi: 10.21037/qims-21-462 